\title{
Variations of $\mathrm{ABCB} 4$ and $\mathrm{ABCB} 11$ genes are associated with primary intrahepatic stones
}

\author{
SHUGUANG PAN ${ }^{*}$, XIAOWU LI ${ }^{*}$, PENG JIANG, YAN JIANG, LING SHUAI, YU HE and ZHIHUA LI \\ Institute of Hepatobiliary Surgery, Southwest Hospital, Third Military Medical University, \\ Shapingba, Chongqing 400038, P.R. China
}

Received January 17, 2014; Accepted August 11, 2014

DOI: $10.3892 / \mathrm{mmr} .2014 .2645$

\begin{abstract}
Variations of the ABCB4 and ABCB11 genes affect the composition of bile and are associated with cholestasis and cholelithiasis. However, their roles in the formation of primary intrahepatic stones (PIS) remains to be elucidated. The aim of the present study was to determine whether there is an association between PIS and variations in these genes. Exon sequencing was performed in order to analyze the ABCB4 and ABCB11 genes of 176 patients with PIS and 178 healthy subjects. One mutation in ABCB4 (no. 69233, G>A) and two other mutations in ABCB11, reference single nucleotide polymorphism (rs)118109635 and rs497692, were identified in association with $\mathrm{PIS}(\mathrm{P}<0.001, \mathrm{P}=0.04$ and $\mathrm{P}=0.02$, respectively). A synonymous mutation at no. $69233 \mathrm{G}>\mathrm{A}$ was detected in exon 26 of ABCB4 in 23 heterozygous patients with PIS. This mutation was not detected in healthy individuals or in the Single Nucleotide Polymorphism Database. No. 69233 G>A in $\mathrm{ABCB} 4$ was not associated with altered protein expression but with a reduced rate of PIS recurrence $(\mathrm{P}=0.01)$. The missense mutation rs118109635 was located on exon 21 of $\mathrm{ABCB} 11$ and was associated with the increased expression of ABCB11 protein $(\mathrm{P}=0.032)$ as well as altered bile salt export pump function. Another synonymous mutation, rs497692 in exon 24 was reported to decrease ABCB11 protein expression $(\mathrm{P}=0.001)$. In addition, the mutations of $\mathrm{ABCB} 11$ were associated with preoperative jaundice $(\mathrm{P}<0.001$ and $\mathrm{P}=0.03$, respectively). Consistently decreased levels of ABCB11 protein were associated with recurrent episodes of cholangitis $(\mathrm{P}=0.006)$ and preoperative jaundice $(\mathrm{P}=0.015)$. By contrast, ABCB4 expression was not found to be associated with clinical manifestations of PIS.
\end{abstract}

Correspondence to: Professor Zhihua Li, Institute of Hepatobiliary Surgery, Southwest Hospital, Third Military Medical University, 30 Gaotanyan Street, Shapingba, Chongqing 400038, P.R. China E-mail: lizhihuapsg@163.com

*Contributed equally

Key words: primary intrahepatic stone, mutation, expression, ABCB4, ABCB11
In conclusion, the present study demonstrated that ABCB4 and ABCB11 may be promising candidate genes for evaluating the risk of developing PIS. Mutations in these genes have been shown to alter the expression and function of their corresponding proteins and therefore may be associated with the formation of PIS.

\section{Introduction}

Primary intrahepatic stones (PIS) are one of the most prevalent hepatobiliary disorders in China and Japan; in China, $>20 \%$ of PIS patients were found to be located in the South-West of the country $(1,2)$. PIS can significantly affect the health and quality of life of patients and treatment of the disease is complex, requiring multiple surgical interventions (2). Long-term PIS may develop into biliary cirrhosis or cancer of the liver bile duct. The pathogenesis of PIS was reported to be associated with risk factors, including bile stasis, chronic inflammation of the bile ducts, bacterial infection and malnutrition $(3,4)$ as well as environmental factors (5-7). Studies have reported that the variation of two genes, $\mathrm{ABCB} 4$ and $\mathrm{ABCB} 11$, may result in altered bile composition and therefore contribute to the pathogenesis of PIS $(8,9)$.

ABCB4 encodes for a lipid translocator, multidrug resistance protein 3 (MDR3), which transports phosphatidylcholine from the inner to the outer leaflet of the canalicular membrane of hepatocytes (10) and therefore has a crucial role in maintaining normal phosphatidylcholine concentrations in bile. Studies have shown that the hereditary depletion of ABCB4 expression may result in progressive familial intrahepatic cholestasis type 3 (PFIC3) (11) and intrahepatic cholestasis of pregnancy (ICP) (12-19). Furthermore, variants of the ABCB4 gene have been identified and were reported to be associated with cholecystolithiasis (20-21). However, the function of $\mathrm{ABCB} 4$ in PIS remains to be elucidated.

ABCB11 encodes for the bile salt export pump (BSEP), which mediates the canalicular secretion of bile salts from hepatocytes into the bile, representing the primary driving force for the generation of bile salts $(8,22-23)$. ABCB11 gene mutations have been shown to decrease the secretion of bile acid (24) and cause PFIC type 2 (PFIC2) as well as benign recurrent intrahepatic cholestasi type 2 (BRIC2) (25-27). Studies have also confirmed that genetic variation in ABCB11 was associated with the pathogenesis of ICP (27). However, 
an association between ABCB11 and PIS has not yet been established.

Mutations of the ABCB4 and ABCB11 genes affect the composition of bile and were associated with cholestasis and cholelithiasis (17,28-29). Therefore, the hypothesis of the present study was that mutations of these genes may be responsible for the development of PIS. To investigate whether variations in ABCB4 and ABCB11 are associated with PIS, variations in these two genes were analyzed in PIS patients and control subjects using exon sequencing and subsequently evaluated to determine possible associations between these variations and the clinical manifestations of PIS.

\section{Materials and methods}

Liver tissue and blood specimens. Samples were obtained from patients at the Department of Hepatobiliary Surgery at Southwest Hospital of the Third Military Medical University (Chongqing, China) between February 2009 and December 2012. Paraffin-embedded liver tissue blocks and blood specimens were obtained from all 176 patients. Another 62 normal liver tissue blocks were obtained from patients with hepatic hemangioma. A total of 178 healthy controls were recruited at random from the Center for Health Inspection at the same hospital. All patients (excluding patients with hepatic hemangioma) were assessed every 3 months following being discharged using ultrasonography and computed tomography. The occurrence of new stones was considered indicative of a relapse. The median follow-up period was 20 months (range, 3-57 months). During this period, 38 patients experienced recurrences of PIS (21.6\%). The present study was approved by the local ethics committee of the Third Military Medical University following the guidelines of the Declaration of Helsinki.

Inclusion and exclusion criteria of study patients and controls. The diagnostic criteria used to identify patients with PIS were as follows: a) No history of chronic liver disease; b) absence of current infection with hepatitis $\mathrm{A}, \mathrm{B}$ or $\mathrm{C}$ virus and cytomegalovirus; c) no previous liver resection for PIS; d) PIS confirmed using abdominal B-mode ultrasound and computed tomography or magnetic resonance cholangiopancreatography (Siemens., Berlin, Germany); e) exclusion of cholecystolithiasis using abdominal B-mode ultrasound; and f) only patients from the Han Chinese population. Age, gender, and body mass index (BMI) were recorded when patients were recruited to the study.

The 62 patients with hepatic hemangioma were all identified by pathological diagnosis. All control subjects underwent routine laboratory investigations and were not genetically related to any of the patients in the study. Controls were selected according to the following criteria a) PIS was not detected during the health inspection at the time of recruitment; b) ethnicities other than Han Chinese were excluded; and c) health was inspected in the same time-frame as the recruitment of patients.

Immunohistochemistry. BSEP and MDR3 proteins were determined immunohistologically using an immunoperoxidase staining method. Paraffin-embedded tissue blocks were cut into serial sections of 3-5 $\mu \mathrm{m}$, which were then deparaffinized in xylene and rehydrated in a graded ethanol series. Endogenous peroxidase activity was quenched using a 3\% hydrogen peroxide solution for $30 \mathrm{~min}$. Antigens for BSEP and MDR3 were retrieved by heating the tissue sections at $120^{\circ} \mathrm{C}$ for 10 min in citrate buffer $(10 \mathrm{mmol} / \mathrm{l}$; $\mathrm{pH}$ 6.0). Following rinsing in phosphate-buffered saline (PBS) for five minutes, sections were incubated at $37^{\circ} \mathrm{C}$ for $120 \mathrm{~min}$ with antibodies against BSEP (HPA019035; Sigma, St. Louis, MO, USA) or MDR3 (ab111209; Abcam, Cambridge, MA, USA). The sections were then incubated with a secondary antibody for HPA019035 (K5007; Dako, Carpinteria, CA, USA) and ab111209 (PV-9003; ZSGB-BIO, Beijing, China) at $37^{\circ} \mathrm{C}$ for $30 \mathrm{~min}$. Negative controls were prepared by omitting the primary antibodies. Immunoreactivity was detected using a Diaminobenzidine Elite kit (K5007; Dako; Zhongshan Biotechnology Company, Beijing, China) and positive staining was identified as brown staining of immunopositive cells. The sections were dehydrated, cleared and mounted.

The immunostained tissue slides were scored according to stain intensity ( 0 , no stain; 1 , slight staining; 2 , moderate staining; 3, intense staining) multiplied by a distribution score (1, staining of $0-33 \%$; 2 , staining of $33-66 \% ; 3,>66 \%$ staining). The final score was grouped as low expression [negative, zero or low (1-2) scores], medium expression (moderate score, 3-4), or high expression (high score, 6-9) for further non-parametric tests (see Fig. 1). Each immunostained slide was scored by two pathologists in a double-blind manner using a microscope (Olympus, Tokyo, Japan).

Genotyping. Genomic DNA was extracted from peripheral venous blood leucocytes using a Blood Genome DNA Extraction kit (D9081; Takara Bio, Inc., Shiga, Japan) according to the manufacturer's instructions. The concentration of extracted DNA was quantified using spectrophotometry with a nucleic acid analyzer NV3000C (Vastech, Inc., San Jose, CA, USA). 54 primer pairs were designed in order to amplify all 54 exons in ABCB4 and ABCB11 (Table I) using standard PCR methods. DNA polymorphism of the genes of interest was determined in all subjects by direct sequencing of all 54 exons of ABCB4 and ABCB11 using an ABI 3730xl genetic analyzer (Applied Biosystems, Foster City, CA, USA).

Statistical analysis. Genotype frequencies were determined by direct counting. The Chi-square test was used to assess the distribution of gender, the Hardy-Weinberg equilibrium of all SNPs in the control group, the difference of transporter protein expression levels in different genotypic groups and the genotypic distributions in different groups according to clinical classification. Associations between polymorphisms and PIS were estimated by odds ratios (ORs) with a 95\% confidence interval (CI), using an unconditional logistic regression model. The ORs were adjusted for age, gender and BMI. All calculations were performed using Statistical Analysis System software (version 9.0, SAS Institute Inc., Cary, NC, USA). P $<0.05$ was considered to indicate a statistically significant difference between values.

\section{Results}

$A B C B 4$ and $A B C B 11$ gene mutations detected that may be associated with the pathogenesis of PIS. It has previously been shown that ABCB4 was involved in the pathogenesis of intrahepatic cholestasis (11-19) and cholecystolithiasis $(20,21)$. 


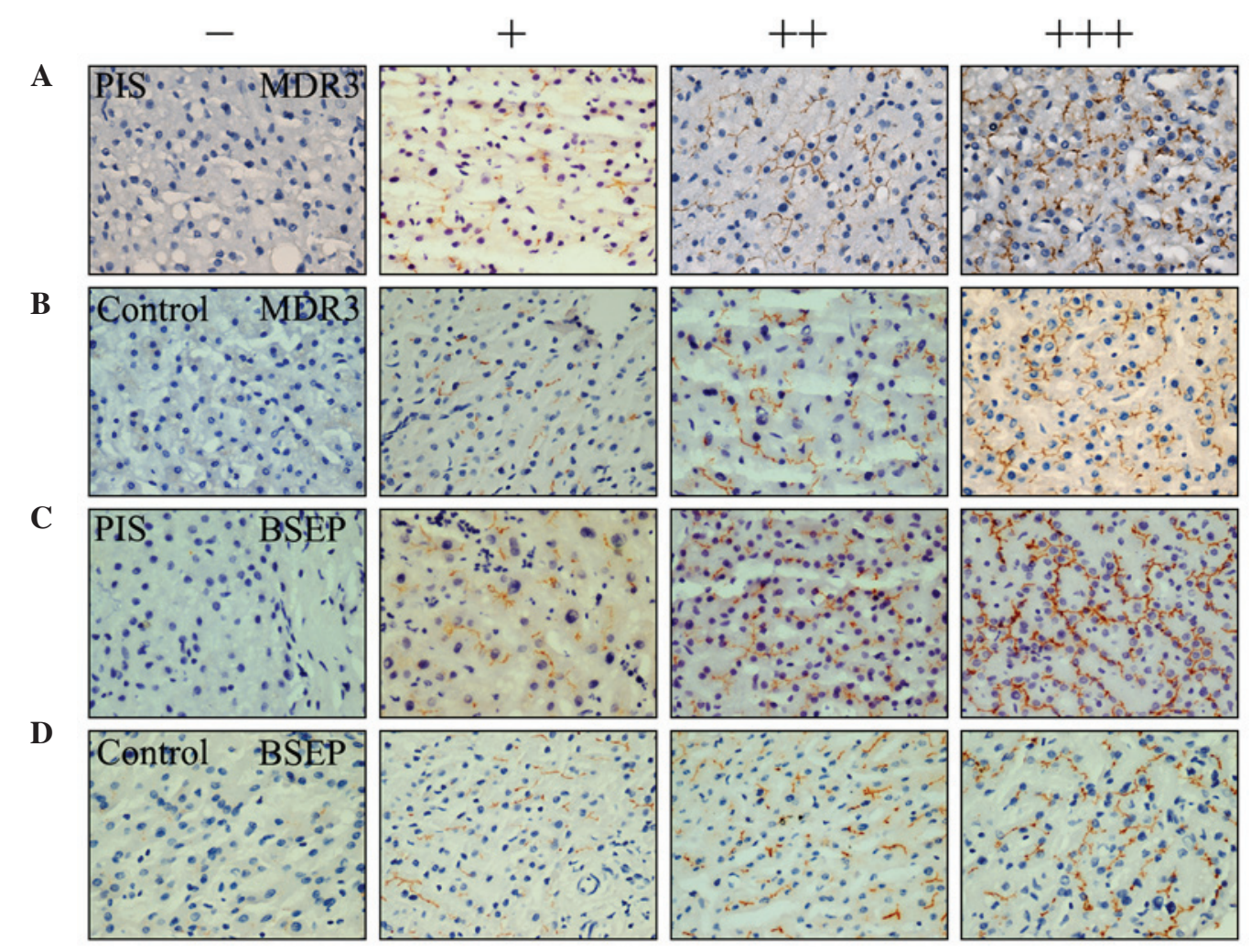

Figure 1. MDR3 and BSEP expression levels, examined by immunohistochemistry in liver tissues of PIS patients and healthy individuals. Immunohistochemical staining of liver tissues of PIS: (A) Left two images indicate low expression of MDR3 and the right two indicate medium and high expression of MDR3, respectively; (B) left two images indicate low expression of MDR3 and the right two indicate medium and high expression of MDR3, respectively; and (C) left two images indicate low expression of BSEP and the right two images indicate medium and high expression of BSEP, respectively. (D) Immunohistochemical staining of liver tissues of healthy controls: left two images indicate low expression of BSEP, right two indicate medium and high expression of BSEP, respectively. (magnification, x400). -, negative or zero score; +, score 1-2; ++ score 3-4; +++, score 6-9; MDR3, multidrug resistance protein 3; BSEP, bile salt export pump; PIS, primary intrahepatic stones.

Exon sequencing of blood samples from 176 patients with PIS and 178 healthy controls was performed in order to analyze the presence of mutations in the ABCB4 gene in patients with PIS (Table II). A missense mutation (no. 22677 G>T; Q151H) was detected in exon 6 of the ABCB4 gene in 20 heterozygous patients with PIS. In addition, a synonymous mutation (no. $69233 \mathrm{G}>\mathrm{A}$ ) was detected in exon 26 in 23 heterozygous patients with PIS. These mutations were not detected in healthy individuals or in the Single Nucleotide Polymorphism Database (dbSNP; gene ID 5244). Two more synonymous mutations were identified (rs1202283 in exon 6 and rs2109505 in exon 8); however, there was no difference in their distributions between the PIS and healthy groups.

ABCB11 has also been associated with the formation of intrahepatic cholestasis $(25,27)$, therefore indicating that it may be involved in the pathogenesis of PIS. The present study analyzed the presence of mutations in the ABCB11 gene in patients with PIS (Table II). As shown in Table II, three nonsynonymous and four synonymous mutations were detected in the ABCB11 gene of PIS patients. In this gene, two missense (rs2287617 and rs2287622) and two synonymous (rs3815675 and rs4148777) mutations were detected in exons 9, 13, 4 and 5, respectively. However, the distributions of allele frequencies for these four mutations were not significantly different between the patients and controls $(\mathrm{P}>0.05)$. Conversely, one missense mutation (rs118109635) and two synonymous mutations (rs2287616 and rs497692) were also detected, respectively, in exons 21,9 , and 24 . The distributions of these allele frequencies were significantly different in PIS patients compared to those in the healthy group $(\mathrm{P}=0.04$, $\mathrm{P}=0.01$ and $\mathrm{P}=0.02$, respectively).

Overall, two novel mutations (no. $22677 \mathrm{G}>\mathrm{T}$ and no. $69233 \mathrm{G}>\mathrm{A}$ ) were identified in the ABCB4 gene of PIS patients. In addition, three mutations in the ABCB11 gene (rs118109635, rs2287616 and rs497692) were found to be associated with the pathogenesis of PIS.

Altered protein expression in PIS patients with $A B C B 4$ and $A B C B 11$ gene mutations. Immunohistochemical staining was used to evaluate whether ABCB4 and $\mathrm{ABCB} 11$ gene mutations in PIS patients affected the expression of their corresponding proteins (Table III). The expression levels of $\mathrm{ABCB} 4$ as well as $\mathrm{ABCB} 11$ proteins were significantly reduced in the liver tissue of patients with PIS compared to those in normal liver tissue. In 20 PIS patients with the GT genotype of the ABCB4 mutation at exon 6 (no. 22677 $\mathrm{G}>\mathrm{T}$ ), the protein expression levels of $\mathrm{ABCB} 4$ were significantly higher in individuals with the GT genotype compared with those with the GG genotype $(\mathrm{P}=0.019)$. By contrast, the ABCB4 mutations at rs1202283, rs2109505 and no. $69233 \mathrm{G}>\mathrm{A}$ did not significantly affect protein expression levels in patients with PIS. 
Table I. Primer pairs for amplification of ABCB4 and ABCB11 coding sequences.

\begin{tabular}{|c|c|c|c|}
\hline Exon & Forward primer & Reverse primer & $\begin{array}{l}\text { Fragment } \\
\text { length (bp) }\end{array}$ \\
\hline \multicolumn{4}{|c|}{$\mathrm{ABCB} 4$} \\
\hline 2 & 5'-GAGAGGGTGTACTTGGTTCTGA-3' & 5'-AGACAAGGAGGAAGAGACATAACA-3' & 600 \\
\hline 3 & 5'-ACTTAGCGACACTGTTAGCATAC-3' & 5'-ATCTCACTATGTTGTCCAGGCT-3' & 537 \\
\hline 4 & 5'-TAGCCGTTCTTTATTACCACAGG-3' & 5'-GGAGTCAACCAGATATCCAAATCA-3' & 590 \\
\hline 5 & 5'-СТССТСТGTGCСТCСТTAGAAG-3' & 5'-GCCATGATGTGTGCATCTTACT-3' & 535 \\
\hline 6 & 5'-GTGGTGGCTCATGCTATAATCC-3' & 5'-TAGGCTATAGATGCTGCTAGACAT-3' & 691 \\
\hline 7 & 5'-GGGAAGATGTTATTCAAGAGGCTA-3' & 5'-GATACAGGAATGAGTCACCACAC-3' & 537 \\
\hline 8 & 5'-GTAGATGTGGAACTTGACAGTGT-3' & 5'-GCCATCAGTAAAGGGTGCTTAT-3' & 554 \\
\hline 9 & 5'-GAATGTTGGTTGGCTTGGAATG-3' & 5'-CTGGACAGTGGAAAGATTCACC-3' & 559 \\
\hline 10 & 5'-AAATAGACCCACTCAGGCAATA-3' & 5'-GATTCTCAGTCTGGCTACAACA-3' & 620 \\
\hline 11 & 5'-ACACACAATCTTCATCTTGGGT-3' & 5'-AGAACACACTTCACAACAAAGC-3' & 455 \\
\hline 12 & 5'-CCTAACTGAGGTTGATTGTTGA-3' & 5'-GAAGGCATTATCCATCGGCATT-3' & 462 \\
\hline 13 & 5'-GGCAAATAATCCTTCCACGAAA-3' & 5'-AGTTGGACAATCTTGCATCTCA-3' & 538 \\
\hline 14 & 5'-GCCTCTGATAAAGTAGTTGTCCTT-3' & 5'-GCACTGGCAAGAATCTTCAATAG-3' & 592 \\
\hline 15 & 5'-CATTCATCCAAGTGCTTAACTGTG-3' & 5'-GTCTCTGGCATTCCATATAACTCA-3' & 615 \\
\hline 16 & 5'-ATCCTTGATTGAGAAGCAGTTAGG-3' & 5'-GCATCTCAGCGTAAAGACTACAT-3' & 572 \\
\hline 17 & 5'-GCTTGTCATTCTCTGCACCTAG-3' & 5'-GTCTGACACCAGTTTCACCAAA-3' & 545 \\
\hline 18 & 5'-TGTAGATGGATACTGGTGAAGGTT-3' & 5'-TTGGTAATGAATGTCTGCTGAGG-3' & 570 \\
\hline 19 & 5'-AGTTGAACATGATTGGTCTGGTG-3' & 5'-GACATCCTGGAGTCTCAATGGA-3' & 503 \\
\hline 20 & 5'-CTACCACAGAGCAGTCAGGAAG-3' & 5'-GGGATAAAGCCAAAGCCAATGA-3' & 504 \\
\hline 21 & 5'-CATTCTGACCAAGAGGCTAAGG-3' & 5'-GGATGACATTTGTCCAAACAGTAG-3' & 591 \\
\hline 22 & 5'-ACCATACTTAGCACAGCCAGAA-3' & 5'-GTCTTGGAGACCTATTCTTGTTGT-3' & 528 \\
\hline 23 & 5'-GGAGTTGAACATTGGAGTCATCA-3' & 5'-CATCTTTGGACACAGGAGTCATTT-3' & 542 \\
\hline 24 & 5'-GGAAGTCACAGTAGTCCTAAGAA-3' & 5'-ATGTCAGTCAAGTTGCCCAAAT-3' & 589 \\
\hline 25 & 5'-СТСТСАССТТСАТТTCACACCAT-3' & 5'-GAGCTAGACTGAATTCCAGACATT-3' & 580 \\
\hline 26 & 5'-TTGCCAGTTAGACCAAGATAGG-3' & 5'-AAGTGCCTTGTCCAAGTTGTTA-3' & 549 \\
\hline 27 & 5'-GAACTGTCAACTGTTAAGCAAC-3' & 5'-CCAACATAATGAAACCCTGTCTC-3' & 532 \\
\hline 28 & 5'-GCATGGGAACCCATTTGTGTTA-3' & 5'-ACAGGTGTCACTTCTAACTCTCA-3' & 607 \\
\hline \multicolumn{4}{|c|}{$\mathrm{ABCB} 11$} \\
\hline 2 & 5'-GGCTCTTTCAGGGAGTTATTAACC-3' & 5'-ACTTGACCAGCTTGTCCTACTT-3' & 335 \\
\hline 3 & 5'-AGAGACAATATGAGCAGGAAGA-3' & 5'-CTGCTTTGTGCCTTTGATATGA-3' & 266 \\
\hline 4 & 5'-TCTGTGAATCGCTAGTGAACCT-3' & 5'-ACACCCACTGCCATAAATCAAC-3' & 313 \\
\hline 5 & 5'-ATACGAACTCTGCCACTCAATT-3' & 5'-GTTAGATACCACTCCAGCTCAG-3' & 492 \\
\hline 6 & 5'-AATGTAATCTCTGGTGGCTTGA-3' & 5'-TGTAGTTCTTAGGGCTTCTGAT-3' & 262 \\
\hline 7 & 5'-CTTAGTTCCCAAGAAGAGGCATT-3' & 5'-CACACCAAATTGCAGTACCTTG-3' & 487 \\
\hline 8 & 5'-GAGAGGCTGTTAATGCTATCCA-3' & 5'-TGTTGCTAACTGTACTCAGGAA-3' & 410 \\
\hline 9 & 5'-TCTTCCTCCTGTCAATGATGTTAC-3' & 5'-ATTACTCTGCTTAGCTCCСТCTT-3' & 412 \\
\hline 10 & 5'-TGCTCTGTGTTTGCGATGATTT-3' & 5'-TGTTTCCACAGACAGACTCCATA-3' & 435 \\
\hline 11 & 5'-TCTCTGCGTTAACATGGAAGAC-3' & 5'-CAAGAGCGAAACTCCATCTCAA-3' & 418 \\
\hline 12 & 5'-GCAGAGATACGCCAAAGATGTT-3' & 5'-AAGACACCTCCATTCCCTATTACT-3' & 337 \\
\hline 13 & 5'-CACAGACACCGAGTATCAACAC-3' & 5'-CCAGGACAGTCTCAATGTATGC-3' & 332 \\
\hline 14 & 5'-TTTCTGCCCATTGGTCAAGTAT-3' & 5'-СТСТTAGTTTCTCCCAGGAATGTA-3' & 331 \\
\hline 15 & 5'-GATCACTGTCAGAAGCCATCAA-3' & 5'-ТАТСААСТАСТСССАТСССТСС-3' & 336 \\
\hline 16 & 5'-TCTAATGTCTGCACAGCCTATT-3' & 5'-GTTGGGAGAACAGTGAGTATTGA-3' & 441 \\
\hline 17 & 5'-TTGCTACTTCTGATGGACTTCTC-3' & 5'-AGGATTAGGACTACAGAGGACTC-3' & 437 \\
\hline 18 & 5'-AACTTGGACACCAGTTGATCCT-3' & 5'-TAGTCTGACTTGAAACACTGCTAG-3' & 300 \\
\hline 19 & 5'-CCATATCCCATAGACATTTGAGGT-3' & 5'-ATGAGAAGAAGAAAGCTAGTCCAG-3' & 335 \\
\hline 20 & 5'-CCACCAGAATGATACATTTCCTAC-3' & 5'-TGAAGAGGGAGATGTTAGAGAA-3' & 405 \\
\hline 21 & 5'-GCAATGGGCTGTGTATCTCTTT-3' & 5'-GTCAGTGTTAGAAGCAGTGGAA-3' & 444 \\
\hline 22 & 5'-TCTGAGACGGGTTGATTGCTTT-3' & 5'-GCTTCCTTCAGTCTCTTCGTACTA-3' & 331 \\
\hline 23 & 5'-CCACTGAAATGTCACGAAAGGA-3' & 5'-TGGAGACAGAAGAATACACAGAAG-3' & 524 \\
\hline 24 & 5'-ATGCTTGTTCAGTCCTCTTCTT-3' & 5'-CCTGTGTCCATGTGTTCTGTT-3' & 560 \\
\hline 25 & 5'-TGAAGGTATCTCAAGCAGGGATT-3' & 5'-AAAGTGAGTCTGGCAAAGCAAA-3' & 397 \\
\hline 26 & 5'-TTAGCCTTGGGATTGTTAGTCTG-3' & 5'-CACTCTGGTCATTCTACTTCTCC-3' & 402 \\
\hline 27 & 5'-GAGGAGACCTTGACATGAGTTC-3' & 5'-GGTTCCACAAAGTATTGCCAAT-3' & 362 \\
\hline 28 & 5'-GGATTGTTATTCAGGTCGTGTT-3' & 5'-TTAGCTTGGATTCCGATGTAGG-3' & 462 \\
\hline
\end{tabular}


Table II. Distributions of 11 SNPs and association between SNPs and PIS.

\begin{tabular}{|c|c|c|c|c|c|c|c|c|}
\hline Gene & $\begin{array}{l}\text { dbSNP } \\
\text { Reference }\end{array}$ & $\begin{array}{l}\text { Amino } \\
\text { acid } \\
\text { change }\end{array}$ & Genotype $^{a}$ & $\begin{array}{c}\text { Cases }(\%) \\
n=176\end{array}$ & $\begin{array}{c}\text { Controls }(\%) \\
n=178\end{array}$ & $\begin{array}{l}\text { Adjusted OR } \\
(95 \% \mathrm{CI})^{\mathrm{b}}\end{array}$ & P-value & $\begin{array}{l}\text { P for } \\
\text { HWE in } \\
\text { control }\end{array}$ \\
\hline \multicolumn{9}{|c|}{ ABCB4 } \\
\hline & \multirow[t]{4}{*}{ rs1202283 } & \multirow[t]{4}{*}{ Synonymous } & $\mathrm{CC}$ & $67(38.1)$ & $76(42.7)$ & Ref. & Ref. & \multirow[t]{4}{*}{0.58} \\
\hline & & & CT & 79 (44.9) & $78(43.8)$ & $1.23(0.72-2.12)$ & 0.45 & \\
\hline & & & TT & $30(17.0)$ & $24(13.5)$ & $1.31(0.61-2.80)$ & 0.48 & \\
\hline & & & $\mathrm{CT}+\mathrm{TT}$ & $109(61.9)$ & $103(57.3)$ & $1.25(0.75-2.08)$ & 0.38 & \\
\hline & \multirow[t]{4}{*}{ no. $22677^{\mathrm{c}}$} & \multirow[t]{4}{*}{ Q151H } & GG & $156(88.6)$ & $178(100.0)$ & Ref. & Ref. & \multirow[t]{4}{*}{ NA } \\
\hline & & & GT & $20(11.4)$ & $0(0.0)$ & NA & $<0.001^{\mathrm{e}}$ & \\
\hline & & & TT & $0(0.0)$ & $0(0.0)$ & NA & NA & \\
\hline & & & $\mathrm{GT}+\mathrm{TT}$ & $20(11.4)$ & $0(0.0)$ & NA & $<0.001^{\mathrm{e}}$ & \\
\hline & \multirow[t]{4}{*}{ rs2109505 } & \multirow[t]{4}{*}{ Synonymous } & AA & $108(61.4)$ & $108(60.7)$ & Ref. & Ref. & \multirow[t]{4}{*}{0.09} \\
\hline & & & AT & $55(31.3)$ & $56(31.5)$ & $1.07(0.62-1.85)$ & 0.82 & \\
\hline & & & TT & $13(7.4)$ & $14(7.9)$ & $0.74(0.29-1.86)$ & 0.52 & \\
\hline & & & $\mathrm{AT}+\mathrm{TT}$ & $68(38.6)$ & $70(39.3)$ & $0.99(0.59-1.64)$ & 0.96 & \\
\hline & \multirow[t]{4}{*}{ no. $69233^{c}$} & \multirow[t]{4}{*}{ Synonymous } & GG & $153(86.9)$ & $178(100.0)$ & Ref. & Ref. & \multirow[t]{4}{*}{ NA } \\
\hline & & & GA & $23(13.1)$ & $0(0.0)$ & NA & $<0.001^{\mathrm{e}}$ & \\
\hline & & & AA & $0(0.0)$ & $0(0.0)$ & NA & NA & \\
\hline & & & $\mathrm{GA}+\mathrm{AA}$ & $23(13.1)$ & $0(0.0)$ & NA & $<0.001^{\mathrm{e}}$ & \\
\hline \multicolumn{9}{|c|}{ ABCB 11} \\
\hline & \multirow[t]{5}{*}{ rs3815675 } & \multirow[t]{5}{*}{ Synonymous } & TT & $90(51.1)$ & $89(50.0)$ & Ref. & Ref. & \multirow[t]{5}{*}{0.10} \\
\hline & & & $\mathrm{TC}$ & $73(41.5)$ & 67 (37.6) & $1.19(0.70-2.02)$ & 0.52 & \\
\hline & & & $\mathrm{CC}$ & $13(7.4)$ & $22(12.4)$ & $0.47(0.19-1.16)$ & 0.10 & \\
\hline & & & $\mathrm{CC}$ & $13(7.4)$ & $22(12.4)$ & Ref. & Ref. & \\
\hline & & & $\mathrm{TC}+\mathrm{TT}$ & $163(92.6)$ & $156(87.6)$ & $2.28(0.96-5.43)$ & 0.06 & \\
\hline & \multirow[t]{4}{*}{ rs4148777 } & \multirow[t]{4}{*}{ Synonymous } & $\mathrm{TT}$ & $159(90.3)$ & $161(90.4)$ & Ref. & Ref. & \multirow[t]{4}{*}{0.50} \\
\hline & & & $\mathrm{TC}$ & $16(9.1)$ & $17(9.6)$ & $0.74(0.31-1.79)$ & 0.50 & \\
\hline & & & $\mathrm{CC}$ & $1(0.6)$ & $0(0.0)$ & NA & 0.99 & \\
\hline & & & $\mathrm{TC}+\mathrm{CC}$ & $17(9.7)$ & $17(9.6)$ & $0.76(0.32-1.83)$ & 0.55 & \\
\hline & rs2287616 & Synonymous & $\mathrm{TT}$ & $83(47.2)$ & $94(52.8)$ & Ref. & Ref. & 0.08 \\
\hline & & & $\mathrm{TC}$ & 79 (44.9) & $64(36.0)$ & $1.58(0.92-2.70)$ & 0.07 & \\
\hline & & & $\mathrm{CC}$ & $14(8.0)$ & $20(11.2)$ & $0.40(0.16-0.96)$ & $0.04^{\mathrm{d}}$ & \\
\hline & & & $\mathrm{CC}$ & $14(8.0)$ & $24(11.2)$ & Ref. & Ref. & \\
\hline & & & $\mathrm{TC}+\mathrm{TT}$ & $162(92.0)$ & $154(88.8)$ & $3.06(1.31-7.18)$ & $0.01^{\mathrm{d}}$ & \\
\hline & rs2287617 & R299K & GG & $173(98.3)$ & $176(98.9)$ & Ref. & Ref. & 0.94 \\
\hline & & & GA & $3(1.7)$ & $2(1.1)$ & $0.58(0.06-5.20)$ & 0.62 & \\
\hline & & & AA & $0(0.0)$ & $0(0.0)$ & NA & NA & \\
\hline & & & $\mathrm{GA}+\mathrm{AA}$ & $3(1.7)$ & $2(1.1)$ & $0.58(0.06-5.20)$ & 0.62 & \\
\hline & rs2287622 & V444A & $\mathrm{TT}$ & $16(9.1)$ & $16(9.0)$ & Ref. & Ref. & 0.47 \\
\hline & & & $\mathrm{TC}$ & $78(44.3)$ & $68(38.2)$ & $1.18(0.48-2.89)$ & 0.73 & \\
\hline & & & $\mathrm{CC}$ & $82(46.6)$ & $94(52.8)$ & $0.83(0.34-2.02)$ & 0.68 & \\
\hline & & & $\mathrm{CC}$ & $82(46.6)$ & $94(52.8)$ & Ref. & Ref. & \\
\hline & & & $\mathrm{TC}+\mathrm{TT}$ & $94(53.4)$ & $84(47.2)$ & $1.37(0.83-2.27)$ & 0.22 & \\
\hline & rs118109635 & A865V & $\mathrm{CC}$ & $166(94.3)$ & 177 (99.4) & Ref. & Ref. & 0.97 \\
\hline & & & CT & $10(5.7)$ & $1(0.6)$ & $9.21(1.09-77.72)$ & $0.04^{\mathrm{d}}$ & \\
\hline & & & TT & $0(0.0)$ & $0(0.0)$ & NA & NA & \\
\hline & & & $\mathrm{CT}+\mathrm{TT}$ & $10(5.7)$ & $1(0.6)$ & $9.21(1.09-77.72)$ & $0.04^{\mathrm{d}}$ & \\
\hline & rs497692 & Synonymous & AA & $21(11.9)$ & $40(22.5)$ & Ref. & Ref. & 0.22 \\
\hline & & & $\mathrm{AG}$ & 75 (42.6) & $80(44.9)$ & $1.78(0.87-3.64)$ & 0.12 & \\
\hline & & & GG & $80(45.5)$ & $58(32.6)$ & $2.43(1.17-5.06)$ & $0.02^{\mathrm{d}}$ & \\
\hline & & & $\mathrm{AG}+\mathrm{GG}$ & $155(88.1)$ & $138(77.5)$ & $2.06(1.05-4.01)$ & $0.04^{\mathrm{d}}$ & \\
\hline
\end{tabular}

${ }^{a}$ The reference alleles were determined following the ancestral alleles in dbSNP, except for loci nos. 22677 and 69233. The common alleles in the two loci were defined as referent alleles. ${ }^{b}$ The position of SNP in DNA sequence from GenBank, Gene ID for ABCB4: 5244. ${ }^{\mathrm{C}}$ Adjusted for age, gender and body mass index. ${ }^{\mathrm{d}} \mathrm{P}<0.05$; ${ }^{\mathrm{e}} \mathrm{P}<0.01$. HWE, Hardy-Weinberg Equilibrium; CI, confidence interval; OR, odds Ratio; Ref., reference; NA, not available; dbSNP, Single Nucleotide Polymorphism Database; PIS, primary intrahepatic stones. 
Table III. Association between SNPs and protein expression.

\begin{tabular}{|c|c|c|c|c|c|}
\hline SNP & $\begin{array}{c}\text { Cases, } \\
\mathrm{n}(\%)\end{array}$ & $\begin{array}{c}\text { Low } \\
\text { expression, } \\
n(\%)\end{array}$ & $\begin{array}{c}\text { Medium } \\
\text { expression, } \\
\mathrm{n}(\%)\end{array}$ & $\begin{array}{c}\text { High } \\
\text { expression, } \\
\text { n }(\%)\end{array}$ & $\mathrm{P}$ \\
\hline $\begin{array}{l}\text { 1) } \mathrm{ABCB} 4 \\
\text { (MDR3 Protein) }\end{array}$ & $\mathrm{n}=176$ & $\mathrm{n}=120$ & $\mathrm{n}=39$ & $\mathrm{n}=17$ & \\
\hline Alleles (2n) & $2 n=352(100)$ & $2 \mathrm{n}=240(100 \%)$ & $2 \mathrm{n}=78(100 \%)$ & $2 \mathrm{n}=34(100 \%)$ & \\
\hline $\begin{array}{l}\text { a) rs } 1202283 \\
\text { (Synonymous) } \\
\text { Genotypes: }\end{array}$ & & & & & 0.618 \\
\hline $\mathrm{CC}$ & $67(100)$ & $48(72)$ & 13 (19) & $6(9)$ & \\
\hline $\mathrm{CT}$ & 79 (100) & $55(70)$ & $16(20)$ & $8(10)$ & \\
\hline TT & $30(100)$ & $17(57)$ & $10(33)$ & $3(10)$ & \\
\hline $\begin{array}{l}\text { b) no. } 22677 \\
(\mathrm{Q} 151 \mathrm{H}) \\
\text { Genotypes: }\end{array}$ & & & & & $0.019^{\mathrm{a}}$ \\
\hline GG & $156(100)$ & $111(71)$ & $33(21)$ & $12(8)$ & \\
\hline GT & $20(100)$ & $9(45)$ & $6(30)$ & $5(25)$ & \\
\hline $\mathrm{TT}$ & 0 & 0 & 0 & 0 & \\
\hline $\begin{array}{l}\text { c) rs2109505 } \\
\text { (Synonymous) } \\
\text { Genotypes: }\end{array}$ & & & & & 0.839 \\
\hline AA & $108(100)$ & $72(67)$ & $24(22)$ & $12(11)$ & \\
\hline AT & $55(100)$ & $40(73)$ & $11(20)$ & $4(7)$ & \\
\hline $\mathrm{TT}$ & $13(100)$ & $8(62)$ & $4(31)$ & $1(7)$ & \\
\hline $\begin{array}{c}\text { d) no. } 69233 \\
\text { (Synonymous) } \\
\text { Genotypes: }\end{array}$ & & & & & 0.063 \\
\hline GG & $153(100)$ & $104(68)$ & $32(21)$ & $17(11)$ & \\
\hline GA & $23(100)$ & $16(70)$ & $7(30)$ & 0 & \\
\hline $\mathrm{AA}$ & 0 & 0 & 0 & 0 & \\
\hline $\begin{array}{l}\text { 2) ABCB11 } \\
\text { (BSEP Protein) }\end{array}$ & $n=176$ & $\mathrm{n}=112$ & $\mathrm{n}=36$ & $\mathrm{n}=28$ & \\
\hline Alleles (2n) & & $2 n=224(100)$ & $2 \mathrm{n}=72(100)$ & $2 n=56(100)$ & \\
\hline $\begin{array}{l}\text { a) rs3815675 } \\
\text { (Synonymous) } \\
\text { Genotypes: }\end{array}$ & & & & & 0.089 \\
\hline TT & $90(100)$ & $57(63)$ & $18(20)$ & $15(17)$ & \\
\hline $\mathrm{TC}$ & 73 (100) & $46(63)$ & $18(25)$ & $9(12)$ & \\
\hline $\mathrm{CC}$ & 13 (100) & $9(69)$ & 0 & $4(31)$ & \\
\hline $\begin{array}{l}\text { b) rs4148777 } \\
\text { (Synonymous) } \\
\text { Genotypes: }\end{array}$ & & & & & 0.510 \\
\hline $\mathrm{TT}$ & $159(100)$ & $102(64)$ & $32(20)$ & $25(16)$ & \\
\hline $\mathrm{TC}$ & $16(100)$ & $10(62)$ & $3(19)$ & 3 (19) & \\
\hline $\mathrm{CC}$ & $1(100)$ & 0 & $1(100)$ & 0 & \\
\hline $\begin{array}{l}\text { c) rs2287616 } \\
\text { (Synonymous) } \\
\text { Genotypes: }\end{array}$ & & & & & 0.088 \\
\hline TT & 83 (100) & $50(60)$ & $19(23)$ & $14(17)$ & \\
\hline $\mathrm{TC}$ & 79 (100) & $52(66)$ & $17(22)$ & $10(13)$ & \\
\hline $\mathrm{CC}$ & $14(100)$ & $10(71)$ & 0 & $4(29)$ & \\
\hline
\end{tabular}


Table III continued.

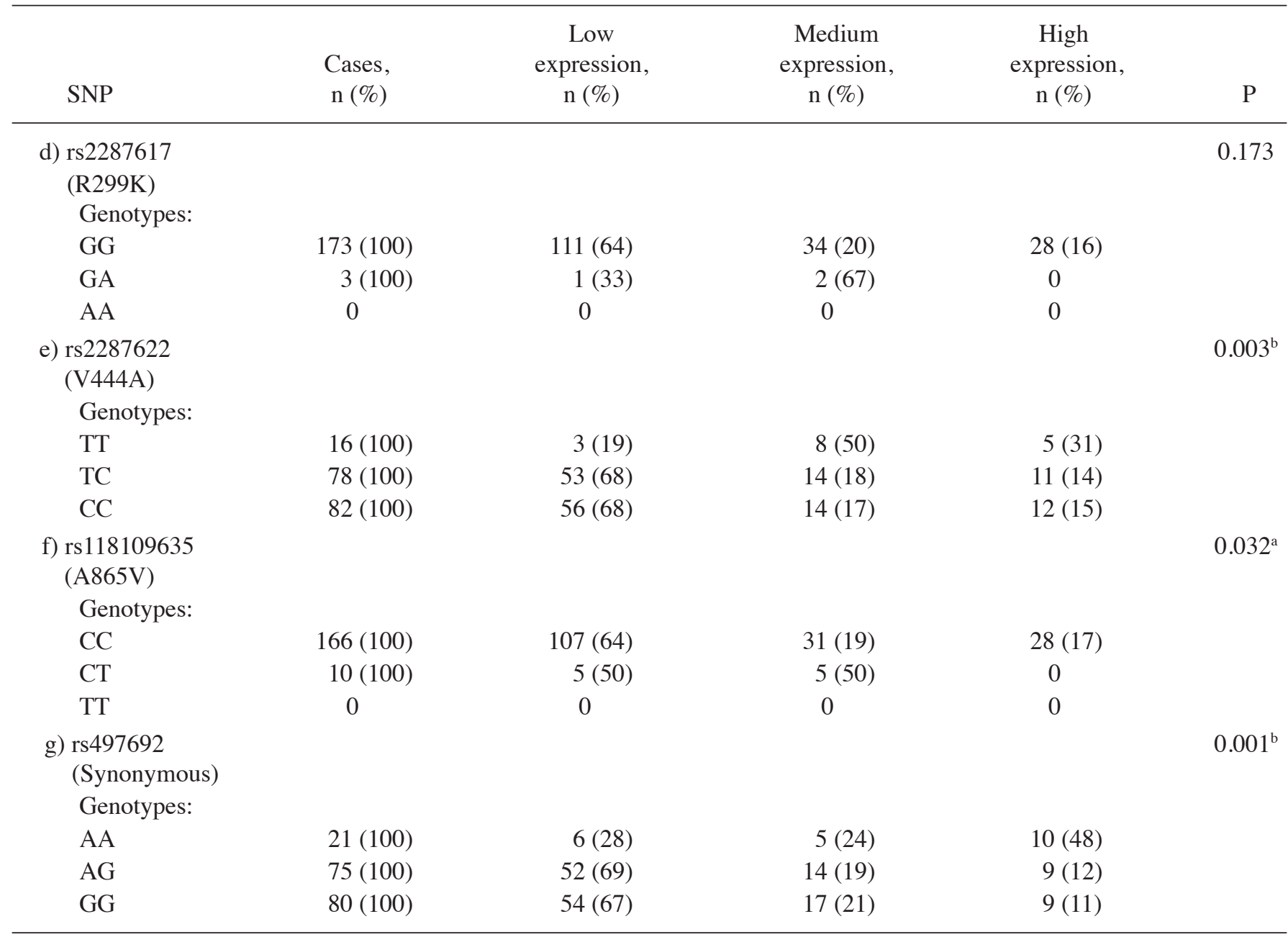

${ }^{\mathrm{a}} \mathrm{P}<0.05 ;{ }^{\mathrm{b}} \mathrm{P}<0.01$, genotype distributions in the protein expression levels were significantly different. SNP, single nucleotide polymorphism.

Table IV. MDR3 and BSEP expression levels were significantly different between cases and controls.

\begin{tabular}{|c|c|c|c|c|c|c|c|c|}
\hline \multirow[b]{2}{*}{ Group } & \multicolumn{4}{|c|}{ MDR3 expression } & \multicolumn{4}{|c|}{ BSEP expression } \\
\hline & Low & Medium & High & P-value & Low & Medium & High & P-value \\
\hline Cases, n (\%) & $120(68)$ & $39(22)$ & $17(10)$ & $<0.001$ & $112(64)$ & $36(20)$ & $28(16)$ & $<0.001$ \\
\hline Control, n (\%) & $22(36)$ & $21(34)$ & $19(30)$ & & $17(27)$ & $15(24)$ & $30(48)$ & \\
\hline
\end{tabular}

MDR3, multidrug resistance protein 3; BSEP, bile salt export pump.

In 10 PIS patients with the ABCB11 CT genotype at exon 21 (rs118109635 $\mathrm{C}>\mathrm{T}$ ), the protein expression levels of $\mathrm{ABCB} 11$ were increased compared to those with the $\mathrm{CC}$ genotype $(\mathrm{P}=0.032)$. By contrast, the protein expression levels of ABCB11 were decreased in individuals with the rs2287622 TC or CC genotype, as well as those with the rs497692 AG or GG genotype compared to patients with wild type $\mathrm{ABCB} 11$ gene expression $(\mathrm{P}=0.003$ and $\mathrm{P}=0.001$, respectively). However, the ABCB11 mutations, rs3815675, rs4148777, rs2287616 and rs2287617, did not alter protein expression levels.
These results therefore indicated that the GT genotype at no. $22677 \mathrm{G}>\mathrm{T}$ of the ABCB4 gene may lead to increased protein expression and the ABCB11 gene mutations rs118109635, rs2287622 and rs497692 may also influence protein expression.

Mutations in $A B C B 4$ and $A B C B 11$ correlate with recurrence of $P I S$, cholangitis and preoperative jaundice. The associations between the clinical manifestations of PIS and mutations of ABCB4 and ABCB11 were analyzed. As shown in Table $\mathrm{V}$, for mutations of ABCB4, 


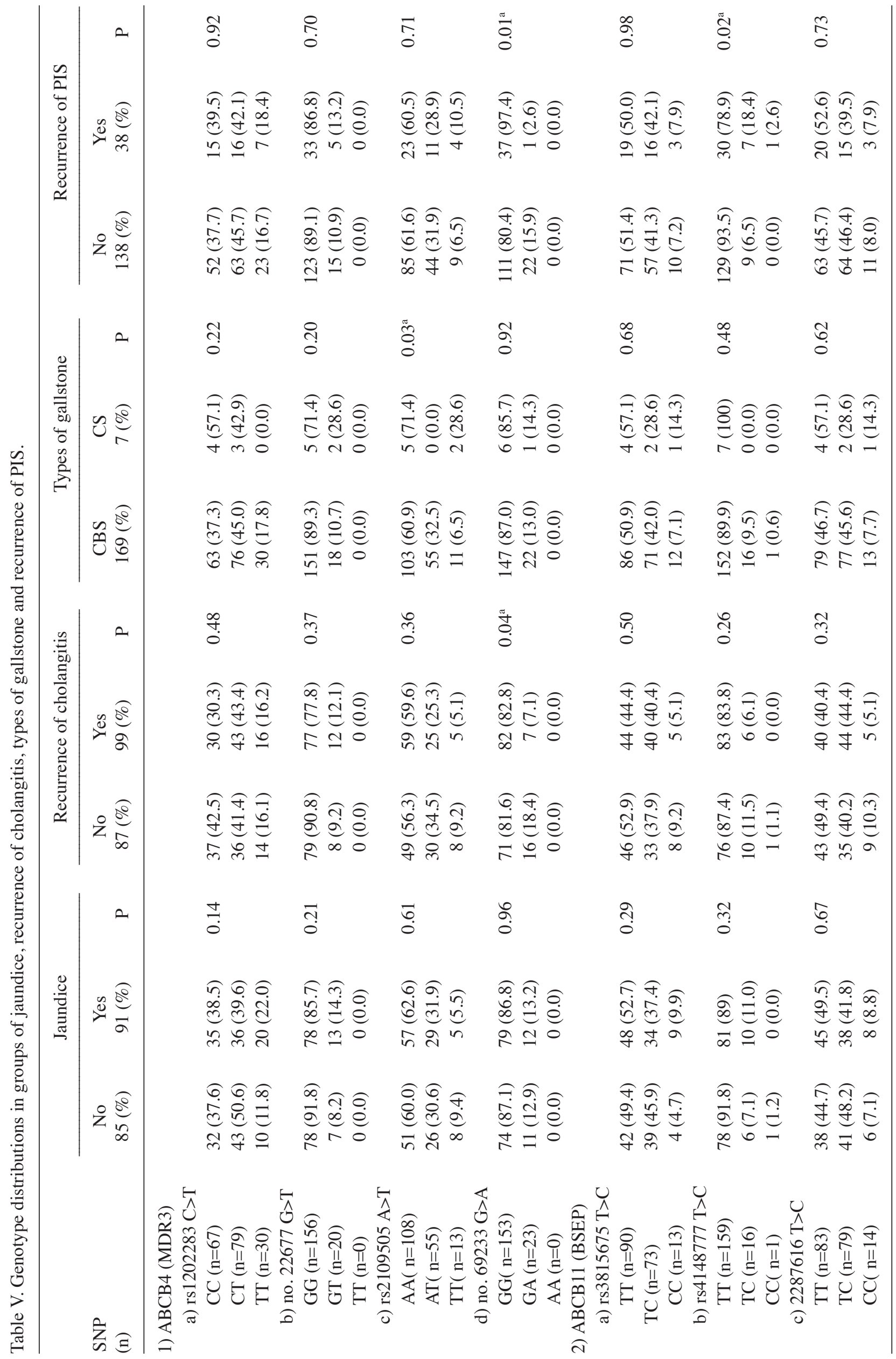




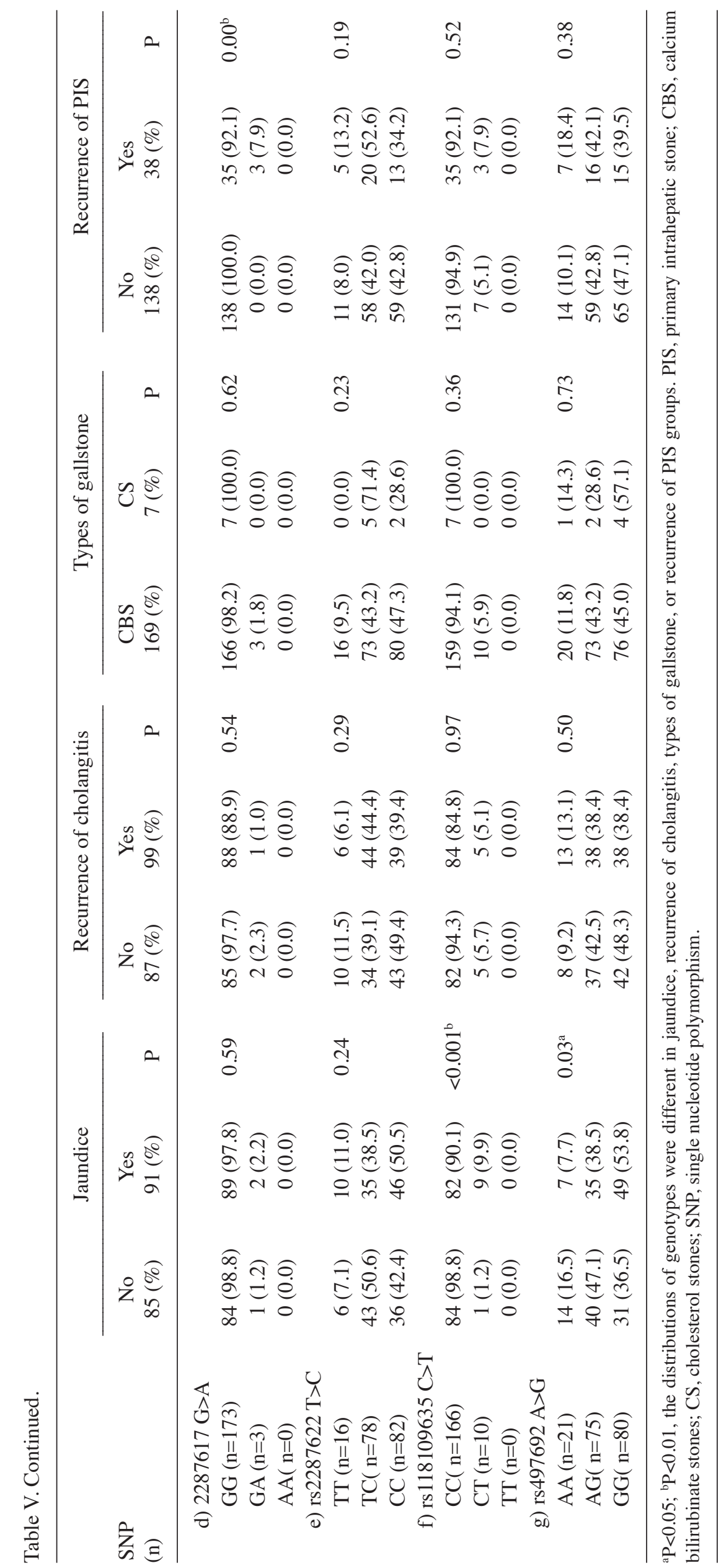


Table VI. BSEP expression levels in groups of gender, preoperative jaundice, recurrence of cholangitis.

\begin{tabular}{|c|c|c|c|c|}
\hline & \multicolumn{3}{|c|}{ BSEP Expression } & \multirow[b]{2}{*}{ P-value } \\
\hline & Low n=112 (\%) & Medium n=36 (\%) & High $n=28(\%)$ & \\
\hline Gender & & & & $0.016^{\mathrm{a}}$ \\
\hline Male $(n=50)$ & $25(50)$ & $17(34)$ & $8(16)$ & \\
\hline Female $(n=126)$ & 87 (69) & $19(15)$ & $20(16)$ & \\
\hline Preoperative jaundice & & & & $0.015^{\mathrm{a}}$ \\
\hline No $(n=85)$ & $58(68)$ & $10(12)$ & $17(20)$ & \\
\hline Yes $(n=91)$ & $54(59)$ & $26(29)$ & $11(12)$ & \\
\hline Recurrence of cholangitis & & & & $0.006^{\mathrm{b}}$ \\
\hline No $(n=87)$ & $53(61)$ & $13(15)$ & $21(24)$ & \\
\hline Yes $(n=89)$ & $59(66)$ & $23(26)$ & $7(8)$ & \\
\hline
\end{tabular}

rs2109505 was associated with types of gallstone $(\mathrm{P}=0.03)$ and no. $69233 \mathrm{G}>\mathrm{A}$ was associated with the recurrence of $\mathrm{PIS}(\mathrm{P}=0.01)$ and recurrent episodes of cholangitis $(\mathrm{P}=0.04)$. Similarly, ABCB11 mutations rs4148777 and rs2287617 were associated with the recurrence of PIS $(\mathrm{P}=0.02, \mathrm{P}<0.001$, respectively); furthermore, rs118109635 and rs497692 mutations of ABCB11 were associated with preoperative jaundice $(\mathrm{P}<0.001$ and $\mathrm{P}=0.03$, respectively). These results suggested that mutations in $\mathrm{ABCB} 4$ and $\mathrm{ABCB} 11$ may be associated with the recurrence of PIS, cholangitis and preoperative jaundice.

Decreased expression of $A B C B 11$ protein is associated with cholangitis and jaundice. Further analysis of the associations between protein expression levels of ABCB11 and the clinical manifestations of PIS revealed an increased recurrence of cholangitis $(\mathrm{P}=0.006)$ and preoperative jaundice $(\mathrm{P}=0.015)$ when ABCB11 protein expression was decreased (Table VI). In addition, it was observed that decreased ABCB11 protein expression was associated with gender, as significantly more females had decreased BSEP expression $(\mathrm{P}=0.016)$. By contrast, the expression of $\mathrm{ABCB} 4$ protein was not associated with the clinical characteristics of PIS. This therefore indicated that altered expression of the ABCB11 protein may be indicative of an increased risk of cholangitis and preoperative jaundice.

\section{Discussion}

The present study identified one novel mutation in ABCB4 (no. $69233 \mathrm{G}>\mathrm{A}$ ) and two novel mutations in ABCB11 (rs118109635 and rs497692) associated with the risk of PIS pathogenesis. In addition, these mutations in ABCB4 and ABCB11 were associated with the recurrence of PIS, cholangitis and preoperative jaundice. Altered expression of the ABCB11 protein was found to be associated with the risk of cholangitis and preoperative jaundice, whereas the expression of ABCB4 was not associated with the clinical characteristics of PIS. By contrast, BSEP and MDR3 levels were decreased in the liver tissue of PIS patients compared to those in normal liver tissue.
Studies have indicated that the formation of intrahepatic stones was attributable to the downregulation of bile acid-synthesis or defective secretion of phospholipids in the liver $(30,31)$. This therefore implied that ABCB4 and ABCB11 may represent promising candidate genes for evaluating the risks of PIS pathogenesis. The present study identified two mutations in ABCB4, not previously reported in the dbSNP, in patients with PIS. The mutation at no. $22677 \mathrm{G}>\mathrm{T}$ was a missense mutation that altered the amino acid sequence. A synonymous mutation (no. $69233 \mathrm{G}>\mathrm{A}$ ) was found to be located at the boundary of the coding region and an intron, suggesting that this mutation may affect the splicing process, resulting in an abnormal messenger RNA (mRNA) transcript. Three mutations were detected in the ABCB11 gene of PIS patients. One was a missense mutation ( $r 118109635 \mathrm{C}>\mathrm{T}$ ), and the other two were synonymous mutations (rs497692 and rs497616). The rs118109635 mutation has previously been reported to be associated with transient neonatal cholestasis $(32,33)$; and the rs497692 mutation was reported to result in defects at either the protein level, mRNA level or a combination of the two, which significantly contributed to BSEP deficiency (34). In addition, the rs497692 mutation was observed in patients with primary biliary cirrhosis and ICP $(35,36)$. To the best of our knowledge, the rs497616 mutation has not been reported to be associated with any disease. The present study performed computational functional analysis of rs497616, and the results indicated that this mutation altered the mRNA splicing process (Table VII). However, further studies are required in order to confirm the association of this functional change with the risk of developing PIS.

ABCB4 and ABCB11 are responsible for the biliary secretion of phosphatidylcholine and bile acids, respectively. Mutations of these genes may affect the composition of bile and are reported to be associated with cholestasis and cholelithiasis $(17,28,29)$. Therefore, mutations of the ABCB4 or ABCB11 genes may be responsible for the pathogenesis of PIS. The present study reported that PIS patients with ABCB4 and ABCB11 mutations have different clinical prognoses. The mutation of the ABCB4 gene at no. $69233 \mathrm{G}>\mathrm{A}$ was a 


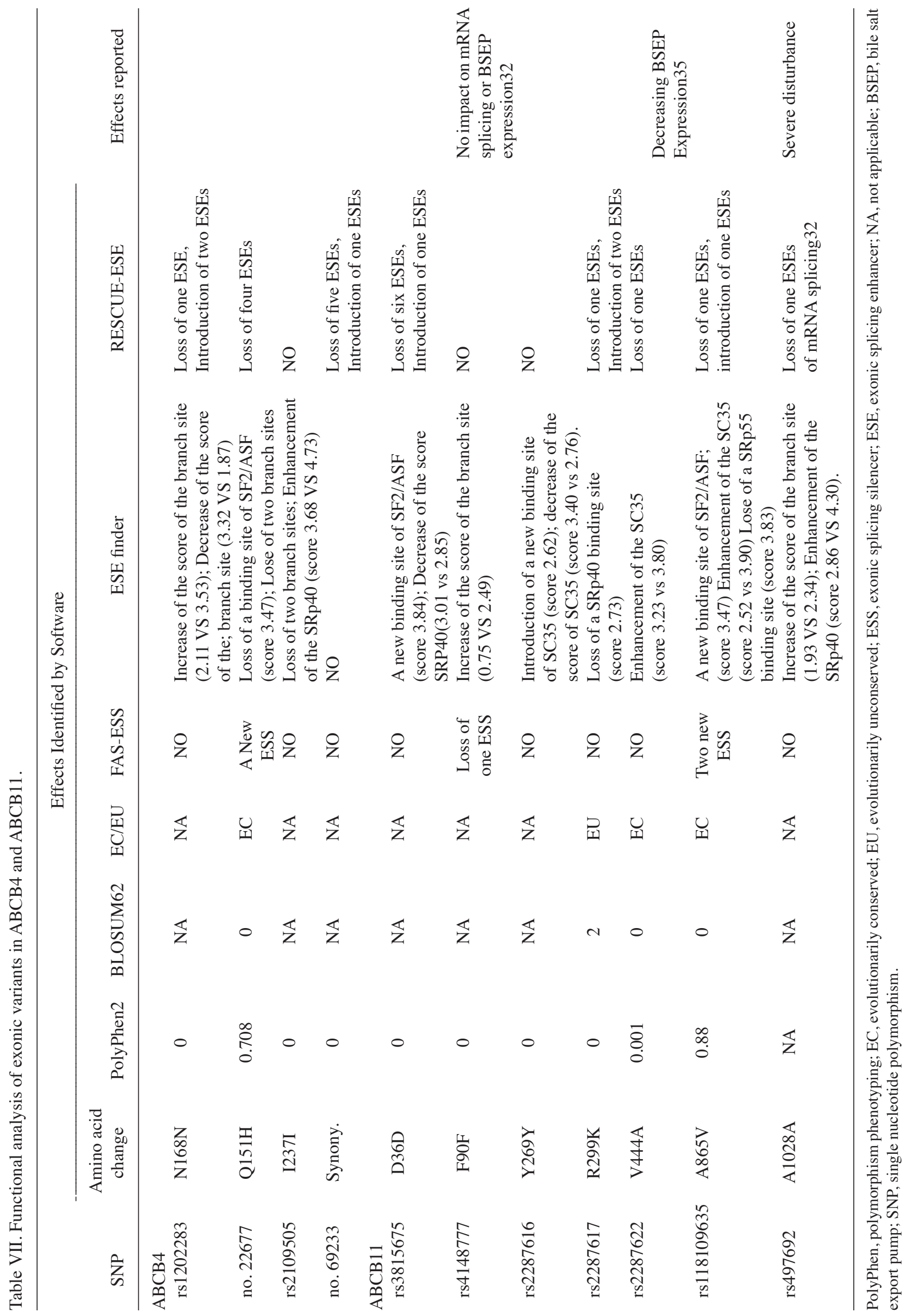


synonymous mutation, which did not alter the levels or function of the corresponding protein; however, this mutation was associated with the decreased recurrence of PIS. Patients with ABCB11 rs118109635 and rs497692 mutations had a significantly increased risk of preoperative jaundice. These results suggested that these mutations may cause BSEP deficiency and increased intrahepatic cholestasis, therefore promoting the formation of PIS. Previous studies on neonatal cholestasis reported comparable results $(32,33)$. Although rs497692 is a synonymous mutation, it resulted in decreased protein expression levels of ABCB11. This result is consistent with observations of Byrne et al (35), which showed that rs497692 was located at the boundary of a coding sequence and an intron, resulting in the loss of one exonic splicing enhancer and the severe disturbance of mRNA splicing. The present study also reported that rs118109635, a missense mutation, was associated with increased levels of ABCB11 protein. Computational functional analysis revealed that this type of mutation may affect the function of BSEP (Table VII). In addition, it has been reported that the most common ABCB11 mutation rs2287622 resulted in decreased ABCB11 protein expression (37); this mutation has been associated with intrahepatic cholestasis (20,38-40), ICP $(35,41)$ and benign recurrent intrahepatic cholestasis (BRIC) (42). This mutation was also detected at high rates in the cohort of PIS patients from the present study (90.9\%) and was associated with decreased levels of ABCB11 protein. However, statistical analysis did not show any differences in the distribution between PIS patients and healthy individuals, nor the association of this mutation with clinical characteristics of patients with PIS. Of note, the ABCB4 missense mutation at no. $22677 \mathrm{G}>\mathrm{T}$ was found to be associated with increased levels of the corresponding protein; however, the functional significance of this remains to be elucidated. Computational functional analysis of no. $22677 \mathrm{G}>\mathrm{T}$ (Table VII) revealed that this type of mutation may affect the function of MDR3. However, the results of the present study showed no association between this mutation and the clinical manifestations of PIS. Therefore, the functional roles of this novel variant in PIS patients requires further characterization. Immunohistochemical analysis demonstrated that decreased expression of ABCB11 protein was associated with cholangitis and preoperative jaundice, whereas ABCB4 expression was not associated with the clinical information of PIS patients. Furthermore, the levels of BSEP and MDR3 were decreased in the liver tissue of PIS patients compared to those in normal liver tissue. The results of the present study, in accordance with previous studies, suggested that the reduction of $\mathrm{ABCB} 4$ and $\mathrm{ABCB} 11$ proteins may alter the composition of bile, including decreased levels of phospholipids (10) and bile salts $(9,43)$. This therefore indicated that the expression of $\mathrm{ABCB} 4$ and $\mathrm{ABCB} 11$ proteins may be responsible for the pathogenesis of PIS.

In conclusion, the results of the present study provided evidence that ABCB4 and $\mathrm{ABCB} 11$ are promising candidate genes, whose mutation may alter the expression and functions of their corresponding proteins and therefore may be indicative of the risk of PIS. This therefore expands the current understanding of the pathogenesis of the PIS and provides the basis for further clinical studies.

\section{Acknowledgements}

This study was supported by grants from the National Natural Science Foundation of China (no. 81270535), the National Science and Technology Major Project of China (no. 2008ZX10002-026) and the Third Military Medical University Science Foundation (no. 2009xlc05).

\section{References}

1. Neuhaus H: Intrahepatic stones: the percutaneous approach. Can J Gastroenterol 13: 467-472, 1999.

2. Mori T, Sugiyama M and Atomi Y: Gallstone disease: Management of intrahepatic stones. Best Prac Res Clin Gastroenterol 20: 1117-1137, 2006.

3. Kaufman HS, Magnuson TH, Lillemoe KD, Frasca P and Pitt HA: The role of bacteria in gallbladder and common duct stone formation. Ann Surg 209: 584-592, 1989.

4. Cetta FM: Bile infection documented as initial event in the pathogenesis of brown pigment biliary stones. Hepatology 6: 482-489, 1986

5. Nakayama F and Koga A: Hepatolithiasis: present status. World J Surg 8: 9-14, 1984.

6. Chang TM and Passaro E Jr: Intrahepatic stones: the Taiwan experience. Am J Surg 146: 241-244, 1983.

7. Nakayama F, Soloway RD, Nakama T, et al: Hepatolithiasis in East Asia. Retrospective study. Dig Dis Sci 31: 21-26, 1986.

8. Oude Elferink RP and Paulusma CC: Function and pathophysiological importance of ABCB4 (MDR3 P-glycoprotein). Pflugers Arch 453: 601-610, 2007.

9. Arrese $\mathbf{M}$ and Ananthanarayanan M: The bile salt export pump: molecular properties, function and regulation. Pflugers Arch 449: 123-131, 2004.

10. Meier Y, Pauli-Magnus C, Zanger UM, et al: Interindividual variability of canalicular ATP-binding-cassette $(\mathrm{ABC})$-transporter expression in human liver. Hepatology 44: 62-74, 2006.

11. Jacquemin E: Role of multidrug resistance 3 deficiency in pediatric and adult liver disease: one gene for three diseases. Semin Liver Dis 21: 551-562, 2001.

12. Jacquemin E, Cresteil D, Manouvrier S, Boute O and Hadchouel M: Heterozygous non-sense mutation of the MDR3 gene in familial intrahepatic cholestasis of pregnancy. Lancet 353: 210-211, 1999.

13. Dixon PH, Weerasekera N, Linton KJ, et al: Heterozygous MDR3 missense mutation associated with intrahepatic cholestasis of pregnancy: evidence for a defect in protein trafficking. Hum Mol Genet 9: 1209-1217, 2000.

14. Lucena JF, Herrero JI, Quiroga J, et al: A multidrug resistance 3 gene mutation causing cholelithiasis, cholestasis of pregnancy, and adulthood biliary cirrhosis. Gastroenterology 124: 1037-1042, 2003

15. Gendrot C, Bacq Y, Brechot MC, Lansac J and Andres C: A second heterozygous MDR3 nonsense mutation associated with intrahepatic cholestasis of pregnancy. J Med Genet 40: e32, 2003.

16. Müllenbach R, Linton KJ, Wiltshire S, et al: ABCB4 gene sequence variation in women with intrahepatic cholestasis of pregnancy. J Med Genet 40: e70, 2003.

17. Pauli-Magnus C, Lang T, Meier Y, et al: Sequence analysis of bile salt export pump (ABCB11) and multidrug resistance P-glycoprotein 3 (ABCB4, MDR3) in patients with intrahepatic cholestasis of pregnancy. Pharmacogenetics 14: 91-102, 2004.

18. Floreani A, Carderi I, Paternoster D, et al: Hepatobiliary phospholipid transporter ABCB4, MDR3 gene variants in a large cohort of Italian women with intrahepatic cholestasis of pregnancy. Dig Liver Dis 40: 366-370, 2008.

19. Schneider G, Paus TC, Kullak-Ublick GA, et al: Linkage between a new splicing site mutation in the MDR3 alias ABCB4 gene and intrahepatic cholestasis of pregnancy. Hepatology 45: 150-158, 2007.

20. Poupon R, Barbu V, Chamouard P, Wendum D, Rosmorduc O and Housset C: Combined features of low phospholipid-associated cholelithiasis and progressive familial intrahepatic cholestasis 3. Liver Int 30: 327-331, 2010.

21. Nakken KE, Labori KJ, Rødningen OK, et al: ABCB4 sequence variations in young adults with cholesterol gallstone disease. Liver Int 29: 743-747, 2009. 
22. Noé J, Stieger B and Meier PJ: Functional expression of the canalicular bile salt export pump of human liver. Gastroenterology 123: 1659-1666, 2002.

23. Byrne JA, Strautnieks SS, Mieli-Vergani G, Higgins CF, Linton KJ and Thompson RJ: The human bile salt export pump: characterization of substrate specificity and identification of inhibitors. Gastroenterology 123: 1649-1658, 2002.

24. Lam P, Pearson CL, Soroka CJ, Xu S, Mennone A and Boyer JL: Levels of plasma membrane expression in progressive and benign mutations of the bile salt export pump (Bsep/Abcb11) correlate with severity of cholestatic diseases. Am J Physiol Cell Physiol 293: C1709-C1716, 2007.

25. van Mil SW, van der Woerd WL, van der Brugge G, et al: Benign recurrent intrahepatic cholestasis type 2 is caused by mutations in ABCB11. Gastroenterology 127: 379-384, 2004.

26. Strautnieks SS, Bull LN, Knisely AS, et al: A gene encoding a liver-specific $\mathrm{ABC}$ transporter is mutated in progressive familial intrahepatic cholestasis. Nat Genet 20: 233-238, 1998.

27. Dixon PH, van Mil SW, Chambers J, et al: Contribution of variant alleles of ABCB11 to susceptibility to intrahepatic cholestasis of pregnancy. Gut 58: 537-544, 2009.

28. Acalovschi M, Tirziu S, Chiorean E, Krawczyk M, Grünhage F and Lammert F: Common variants of ABCB4 and ABCB11 and plasma lipid levels: a study in sib pairs with gallstones, and controls. Lipids 44: 521-526, 2009.

29. Marschall HU, Katsika D, Rudling M and Einarsson C: The genetic background of gallstone formation: an update. Biochem Biophys Res Commun 396: 58-62, 2010.

30. Shoda J, Inada Y and Osuga T: Molecular pathogenesis of hepatolithiasis - a type of low phospholipid-associated cholelithiasis. Front Biosci 11: 669-675, 2006.

31. Tazuma S: Gallstone disease: Epidemiology, pathogenesis, and classification of biliary stones (common bile duct and intrahepatic). Best Pract Res Clin Gastroenterol 20: 1075-1083, 2006.

32. Chen HL, Liu YJ, Su YN, et al: Diagnosis of BSEP/ABCB11 mutations in Asian patients with cholestasis using denaturing high performance liquid chromatography. J Pediatr 153: 825-832, 2008 .

33. Liu LY, Wang XH, Lu Y, Zhu QR and Wang JS: Association of variants of ABCB11 with transient neonatal cholestasis. Pediatr Int 55: 138-144, 2013.
34. Byrne JA, Strautnieks SS, Ihrke G, et al: Missense mutations and single nucleotide polymorphisms in ABCB11 impair bile salt export pump processing and function or disrupt pre-messenger RNA splicing. Hepatology 49: 553-567, 2009.

35. Keitel V, Vogt C, Häussinger D and Kubitz R: Combined mutations of canalicular transporter proteins cause severe intrahepatic cholestasis of pregnancy. Gastroenterology 131: 624-629, 2006.

36. Pauli-Magnus C, Kerb R, Fattinger K, et al: BSEP and MDR3 haplotype structure in healthy Caucasians, primary biliary cirrhosis and primary sclerosing cholangitis. Hepatology 39: 779-791, 2004.

37. Ho RH, Leake BF, Kilkenny DM, et al: Polymorphic variants in the human bile salt export pump (BSEP; ABCB11): functional characterization and interindividual variability. Pharmacogenet Genomics 20: 45-57, 2010.

38. Fattinger K, Funk C, Pantze M, et al: The endothelin antagonist bosentan inhibits the canalicular bile salt export pump: a potential mechanism for hepatic adverse reactions. Clin Pharmacol Ther 69: 223-231, 2001.

39. Eloranta ML, Häkli T, Hiltunen M, Helisalmi S, Punnonen K and Heinonen S: Association of single nucleotide polymorphisms of the bile salt export pump gene with intrahepatic cholestasis of pregnancy. Scand J Gastroenterol 38: 648-652, 2003.

40. Lang C, Meier Y, Stieger B, et al: Mutations and polymorphisms in the bile salt export pump and the multidrug resistance protein 3 associated with drug-induced liver injury. Pharmacogenet Genomics 17: 47-60, 2007.

41. Muehlenberg K, Wiedmann K, Keppeler H, Sauerbruch T and Lammert F: Recurrent intrahepatic cholestasis of pregnancy and chain-like choledocholithiasis in a female patient with stop codon in the ABDC4-gene of the hepatobiliary phospholipid transporter. Z Gastroenterol 46: 48-53, 2008 (In German).

42. Kubitz R, Keitel V, Scheuring S, Köhrer K and Häussinger D: Benign recurrent intrahepatic cholestasis associated with mutations of the bile salt export pump. J Clin Gastroenterol 40: 171-175, 2006.

43. Higuchi $\mathrm{H}$ and Gores GJ: Bile acid regulation of hepatic physiology: IV. Bile acids and death receptors. Am J Physiol Gastrointest Liver Physiol 284: G734-G738, 2003. 